\title{
EFFICIENT ROUTING PROTOCOL TO SUPPORT QOS IN WIRELESS MESH NETWORK
}

\author{
Chems-eddine BEMMOUSSAT ${ }^{1}$, Fedoua DIDI ${ }^{2}$, Mohamed FEHAM ${ }^{3}$ \\ ${ }^{1,3}$ Dept of Telecommunication, Tlemcen University, Tlemcen, Algeria \\ \{chemseddine.benmoussat, m_feham\}@mail.univ-tlemcen.dz \\ ${ }^{2}$ Dept of Computer engineering, Tlemcen University, Tlemcen, Algeria \\ f_didi@mail.univ-tlemcen.dz
}

\begin{abstract}
The wireless mesh network is a new emerging technology that will change the world of industrial networks connectivity to more efficient and profitable. Mesh networks consist of static wireless nodes and mobile customer; have emerged as a key technology for new generation networks. The Quality of Service $(Q O S)$ is designed to promote and support multimedia applications (audio and video), real time. However guarantee of QoS on wireless networks is a difficult problem by comparison at its deployment in a wired IP network. In this paper, we present an efficient routing protocol named as "QoS-Cluster Based Routing Protocol" (Q-CBRP) to support QoS in Wireless Mesh Network.
\end{abstract}

\section{Keywords}

Wireless mesh network, routing protocols, CBRP, QoS.

\section{INTRODUCTION}

Wireless Mesh Networks (WMNs) are one of the key technologies which will dominate wireless networking in the next decade. They will help to realize the long-lasting dream of network connectivity anywhere anytime with simplicity and low cost. Accordingly they will play a major role within the next generation Internet. Their capability for self-organization significantly reduces the complexity of network deployment and maintenance, and thus, requires minimal upfront investment [1].

Wireless mesh networks (WMNs) have emerged as a key technology for next generation wireless networks showing rapid progress and inspiring numerous applications [2].

A wireless mesh network consists of a number of wireless stations (mesh routers MRs) that cover a large area. The nodes communicate with each other in a multi-path, multi-hop fashion via the wireless links to build a cost-effective and easy-configurable wireless backbone for providing Internet connectivity to wireless Mesh clients (M.C) [3]. 


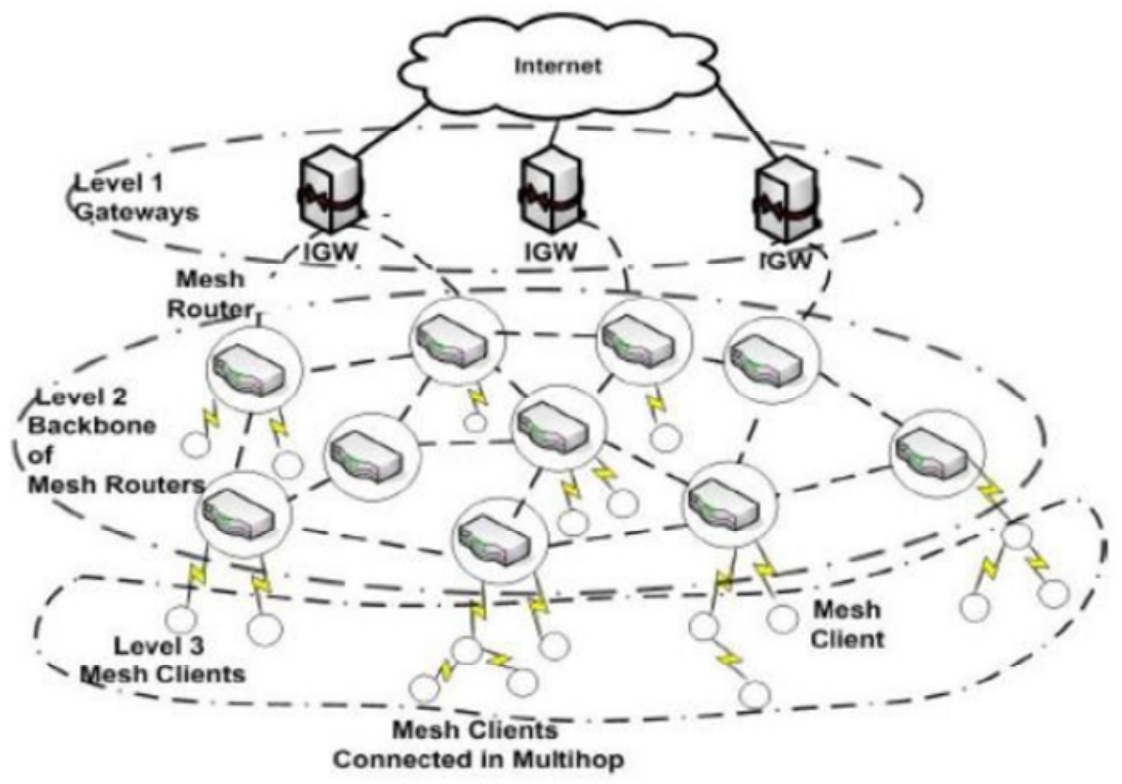

Figure1. Wireless Mesh Network

An example of mesh networks is illustrated in Figure1. In fact the Mesh network is divided into 3 levels

- The first level is generally distinguished gateway nodes for wireless mesh nodes passed to external networks such as Internet, GSM, Wimax...

- The 2nd level is the mesh nodes (wireless AP) that are usually fixed to create the skeleton of 802.11s wireless network and serve the 3rd level.

- The 3rd level is composed of Mesh customers; these customers are often mobile and use services via the $802.11 \mathrm{~s}$.

\section{ROUTING PROTOCOLS}

In general, there are two main types of routing protocols for multi-hop wireless networks: (i) topology-based protocols which need topological information to set up a path between the nodes, (ii) position-based protocols which require some geographical information for the route discovery process. Among the topology-based routing protocols considered here, two distinct categories can be defined:

1) Proactive which maintain the information about the routes to every node all the time sending periodic updates even if the nodes do not communicate with each other, including

DSDV(Destination-Sequenced-Distance Vector) and OLSR (Optimized Link State Routing,) [4]

2) Reactive called also 'on-demand' for which the paths are computed and maintained only when needed, including AODV (Ad-hoc On-demand Distance Vector), and DSR (Dynamic Source Routing). [4].

Short description containing main features of the considered protocols is given below. 
International Journal of Wireless \& Mobile Networks (IJWMN) Vol. 4, No. 5, October 2012

DSDV,it is a modification of Bellman- Ford algorithm implemented in RIP (Routing Information Protocol) adapted for self-configuring networks. Every node maintains its own routing table with the information about network topology and the cost of the links between the nodes.

OLSR, it uses shortest-path algorithm having the access to the routing information whenever it is needed (storing and updating periodically). The optimization idea is based on specific neighborhood detection and Multipoint Relays (MPR-s) selection concept.

AODV, it uses Route Request/ Route Reply (RREQ/RREP) mechanism for route discovery and destination sequence numbers for each route entry like DSDV. This helps detecting outdated routing. Moreover, it keeps track of the next hop instead of the entire route.

DSR, similarly to AODV, it is based on RREQ/RREP packets. However, RREQ gathers the addresses of the 'visited' nodes and maintains information about the whole path from the source to the destination node, not just the next hop. Moreover, the information is stored by every node in a route cache instead of the routing table. [4]

\section{RELATED WORK}

According to the United Nations Consultative Committee for International Telephony and Telegraphy (CCITT) QoS is: "The collective set of service performance which determines the degree of satisfaction of a user of the service". With the increase in technological advancement in the area of wireless network, it becomes mandatory to consider the QoS factors in the routing protocols. To support QoS, information regarding various QoS factors such as delay, bandwidth, cost, loss rate, and error rate in the network should be available and manageable. However, getting and managing these in WMNs is very difficult because of the resource limitations and the complexity associated with the mobility of Mesh users.

The conventional protocols like link-state protocols are not suitable for the multi-hop wireless network as they require that each node has the information about whole of the network which is not possible for large networks. On-demand routing protocols also need flooding of data which will increase the communication overhead.

In order to provide quality of service in the WMNs network the following models have been proposed:

In the beginning of the Mesh network researchers begin to analyze the existing routing protocols.

In [5] the work is divided into two parts: the first part, the compared protocols are: AODV, DSR, DSDV and OLSR, using a fixed topology and other mobile on wireless mesh network with NS -2. The results show that the protocol AODV is the best in terms of delay, throughput and that the DSR is the worst among the four protocols mentioned.

Furthermore, the authors introduced TCP and UDP in the scenarios of the first part, to assess the degree of impact of the transport layer at the network layer. The results confirm that UDP is more interesting than TCP in terms of QoS management.

There is no ideal and the best routing recommendation for WMN. From the protocols studied in this paper, OLSR and AODV should be considered as the ideas worth considering. However, scalability is one of the crucial problems also in this case. One of the solutions is to propose a new routing metric for the existing protocols, use hybrid routing techniques or/and multiple 
International Journal of Wireless \& Mobile Networks (IJWMN) Vol. 4, No. 5, October 2012

radios and interfaces in order to improve performance of the network and provide better capacity of the network

After our previous analysis and existing literature, the routing protocol AODV is most advantageous to ensure QoS, and naturally, lot of works was directed towards the extension of AODV, to improve its performances. It is the aim of the paper [6]. Rate aware routing protocol based on AODV (R-AODV) use minimum network layer transmission time as a performance metric. Nodes will select higher data rate link using R-AODV.

The simulation result indicates that R-AODV can improve the network throughput and decrease network delay.

For specific application like, emergency or search and rescue operations in case of natural disaster, policing and fire fighting military applications such as on the battle field, meeting rooms, sports stadium etc, almost all routing protocols in one way or other, try to converge into shortest path routing. One of the advantages of using shortest path routing is that it is good for overall energy efficiency because energy needed to transmit a packet is directly proportional to path length or number of hops. But the shortest path routing is restricted to use the same set of hops to route the data packets, thus causing some of the heavily loaded nodes and thus causing some of the nodes to die earlier resulting into holes in the network or even worst into partitioning of the network. Thus the need for load balanced routing emerges.

Authors in [7] formulate the problem of routing as a network optimization problem, and present a general LP (linear programming) formulation for modeling the problem. The authors propose the optimized algorithm for known traffic demand and then explain the performance ratio for this. The routing algorithms derived from these formulations usually claim analytical properties such as optimal resource utilization and throughput fairness. The simulation results demonstrate that their statistical problem formulation could effectively incorporate the traffic demand uncertainty in routing optimization, and its algorithm outperforms the algorithm which only considers the static traffic demand. To achieve this objective the problem for congestion has been designed.

Bandwidth overhead and are very important to have a robust network, En efficient routing protocol can solve these problem; we will summarize the recent proposed algorithm.

The goal of the proposed routing protocol [8] is to establish a route from the source to the destination that allows traffic flow within a guaranteed end-to-end latency using the minimum control overhead. The protocol is based on a reliable estimation of wireless link quality and the available bandwidth on a routing path. It also minimizes control overhead by effectively controlling broadcast messages in the network. The QoS-awareness in the protocol is achieved by a robust estimation of the available bandwidth of the wireless channel and a proactive discovery of the routing path by an accurate estimation of the wireless link quality. In addition, the protocol uses the multi-point relay (MPR) nodes to minimize the overhead due to flooding.

The key contributions of the paper are as follows: (i) it exploits the benefits of using MPRs and circular routing to increase the network throughput by reducing the control overhead. (ii) It computes a link quality estimator and utilizes it in route selection. (iii) It provides framework for reliable estimation of available bandwidth in a routing path so that flow admission with guaranteed QoS satisfaction can be made. It also ensures that the number of retransmission required is minimized.

On the forward path, from mesh nodes to Internet nodes, for all mesh nodes only route information for one destination, the gateways, needs to be maintained. However, on the 
International Journal of Wireless \& Mobile Networks (IJWMN) Vol. 4, No. 5, October 2012

backward path from the Internet to mesh nodes, an individual route for every mesh node is required.

In [9], the authors investigate protocols for backward path routing in wireless mesh networks. Using simulation experiments with realistic mobility patterns of pedestrians and cars in cities, they compare three protocols, each of which represents a routing protocol family: (i) AODV with an extension for mesh networks, a reactive routing protocol, (ii) FBR, a proactive routing protocol, and (iii) GSR, a source routing protocol. Their results indicate that FBR has the highest packet delivery ratio but is not scalable to the network size. The extended AODV seems to be neither scalable nor does it achieve a high packet delivery ratio. A good compromise is provided by GSR, which is the most scalable.

Another vision to create a solution to guarantee the bandwidth in WMN is proposed bu Liu et al. Liu et al. [10] proposed an available bandwidth estimation algorithm plus a QoS backup route mechanism to accommodate multimedia traffic flows in mobile wireless mesh networks. Moreover, to validate the correctness of our proposed algorithm, the authors have implemented the algorithm on the campus wireless mesh network testbed. Their implementation and experiments show that their mechanisms can improve the network stability, throughput, and delivery ratio effectively, while decreasing the number of route failure. They implement their proposed algorithms on the testbed through an improved DSR protocol. Their implementation and experiments show that the mechanisms can effectively improve the network stability, throughput, delivery ratio, while decreasing the route invalidation ratio, and can guarantee the fluent transmission of multimedia streams.

In order to support multimedia transmission with QoS requirements, they improve the wireless routing protocol on the testbed with a dynamic ACK mechanism, which is used to balance the throughput and the quality of transmission. Additionally, authors introduce a dynamic mechanism to change the multimedia coding rate dynamically at the source node according to the available bandwidth. Moreover, they also made improvement on the admission control protocol to facilitate an experiment.

The first assertion that we can do, is that, according to the comparative studies results, done to determine what is the best choice between the existing routing algorithms in the state of the art, AODV and OLSR are the best choice by report to others, in terms of QOS.

The second assertion is that several trends have emerged, as follows:

- Extending the traditional routing algorithms such as AODV, DSR, and OLSR, to improve their performances.

- Changing values of the metric, like hybrid or dynamic metric, as bandwidth of links, or end-to-end latency instead of number of hops, for example.

- Propose protocols completely different from those present in the802.11s standard.

- Use of the clustering approach

The mesh network, as is a special case of Ad-hoc networks and MANET networks. These include a new vision of routing protocols based clusters, whose principle is very simple: divide the whole network into several parts, each party will elect a central node, responsible for coordination of routing information between other adjacent nodes, that node is named $\mathrm{CH}$ (Cluster Head), other nodes called its members. Communication in this type of network is simple, any member wishing to transmit, do it through its $\mathrm{CH}$. The latter has a routing table, if 
International Journal of Wireless \& Mobile Networks (IJWMN) Vol. 4, No. 5, October 2012

the destination is internal (in the same group), then the delivery will be direct, if not the $\mathrm{CH}$ sends queries to neighbors to find the right path.

Very recent works have focused on this type of MANET routing. Mukesh Kumar [12] compared a routing protocol named CBRP (Cluster Based Routing Protocol) which gave results much interest as the basic protocols in terms of QoS (delay, throughput) and a good transition to across the MANET.

These comparisons motivate us for our proposed algorithm.

\section{Efficient Cluster-Based Routing Protocol}

In this section, we present the basic idea of the Q-CBRP and its implementation in detail.

Section 3.1 describes the routing process of CBRP briefly. In section 3.2 we discuss about the terminology of Q-CBRP. Sections 3.3 present a functioning of Q-CBRP.

\subsection{Overview of CBRP}

CBRP (Cluster Based Routing Protocol) is an on-demand routing protocol, where the nodes are divided into clusters. It uses clustering's structure for routing protocol.

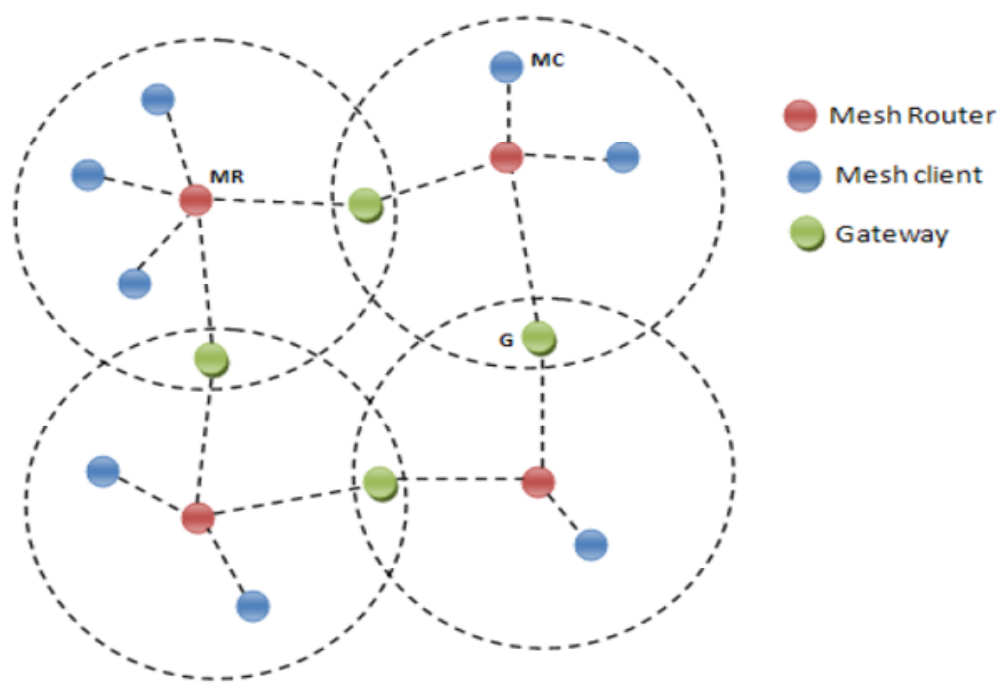

Figure2: WMN with Clustering Architecture

Clustering is a process that divides the network into interconnected substructures, called clusters. Each cluster has a cluster head as coordinator within the substructure. Each cluster head acts as a temporary base station within its zone or cluster and communicates with other cluster heads.

CBRP is designed to be used in Wireless sensor network and mobile ad hoc network. The protocol divides the nodes of the ad hoc network into a number of overlapping or disjoint 2-hop diameter clusters in a distributed manner. Each cluster chooses a head to retain cluster membership information. There are four possible states for the node: Normal, Isolated, Clusterhead $(\mathrm{CH})$ and Gateway. Initially all nodes are in the state of Isolated. Each node maintains the Neighbor table where in the information about the other neighbors nodes is stored; cluster heads have another table (cluster heads neighbor) where include the information about the other 
International Journal of Wireless \& Mobile Networks (IJWMN) Vol. 4, No. 5, October 2012

neighbor cluster heads is stored. [13] The protocol efficiently minimizes the flooding traffic during route discovery and speeds up this process as well.

TABLE 1. Cluster Head Table

\begin{tabular}{|c|c|c|}
\hline $\begin{array}{c}\text { ID_neighbors__ } \\
\text { Clusters }\end{array}$ & $\begin{array}{c}\text { ID_neighbors_ } \\
\text { Gateways }\end{array}$ & ID_members \\
\hline
\end{tabular}

- ID_membres : ID of all members in the same $\mathrm{CH}$

TABLE 2. Gatway Table

ID_CH

TABLE 3. Members Table

\begin{tabular}{|l|l|l|}
\hline ID_Cluster & Status & Link Status \\
\hline
\end{tabular}

- Status of neighboring nodes (Cluster-head, gateway or member)

- Link status (uni-directional or bi-directional)

Route discovery is done by using source routing. In the CBRP only cluster heads are flooded with route request package (RREQ). Gateway nodes receive the RREQs as well, but without broadcasting them. They forward them to the next cluster head. This strategy reduces the network traffic.

Initially, node $\mathrm{S}$ broadcasts a RREQ with unique ID containing the destination's address, the neighboring cluster head(s) including the gateway nodes to reach them and the cluster address list which consists the addresses of the cluster heads forming the route [16].

\subsection{Terminologie for Q-CBRP}

In previous works [15-16], the results show that the protocol CBRP improves QoS in mobile ad-hoc network in general. We didn't stop in this idea; so we study in detail the basic protocol to make improvements to ensure QoS in our Mesh Network.

Our improvements are summarized in two points. First we improve packet header of basic CBRP with more information to have a more complete protocol and the second point we add some fields in routing tables that we will explain in the next.

\begin{tabular}{|c|c|c|c|c|c|}
\hline $\begin{array}{c}\text { Packet } \\
\text { ID }\end{array}$ & $\begin{array}{c}\text { Source } \\
\text { Address }\end{array}$ & $\begin{array}{c}\text { Dest__ } \\
\text { Address }\end{array}$ & $\begin{array}{c}\text { List_of_visited } \\
\text { _node }\end{array}$ & TTL & R(bps) \\
\hline
\end{tabular}

Figure 3. Data packet header

Figure 3 describe our proposal Data Packet Header (DPH), different to DPH in CBRP, where we add two fields in the DPH of original CBRP, the TTL (Time To Live), contains a count of number of intermediate nodes traversed to avoid the packets loop and management of the 
International Journal of Wireless \& Mobile Networks (IJWMN) Vol. 4, No. 5, October 2012

available bandwidth to guarantee QoS $(\mathrm{R})$ it signifies the minimum bandwidth required by a Mesh client to transmit the data.

In our algorithm (Q-CBRP): Cluster Head Table is the same tables in CBRP protocol (Table 1) but an improvement are added in the Gateway Table (Table 2).

Gateway Table maintains the information regarding the gateway node and the available bandwidth over those nodes. We add in Gateway Table an Available Bandwidth, that mean when the data packet is sent to the destination or intermediate node it will reserve the bandwidth required by it. To perform this function of managing bandwidth, admission control mechanism is added where we also block flows when there is not enough bandwidth to avoid packets loss.

TABLE 4. Gateway Table in Q-CBRP

\begin{tabular}{|c|c|c|}
\hline ID_CH & ID_Members & \\
\hline
\end{tabular}

In Q-CBRP, the Member Table maintains the information about its neighboring nodes by broadcasting a Beacon Request Packet.

\subsection{QoS- Cluster Based Routing Protocol for WMN}

Each node in the cluster maintains a table called as Member table (Table 3) containing the address of Neighboring nodes. This table is maintained in the decreasing order of their distance from this particular node. Each node also stores the address of the Cluster-head. Cluster-head also maintains member table as well as it also maintains a gateway table which stores the address of gateway nodes in the decreasing order of distance from the centre head node. This Gateway table stores address as well as the available bandwidth of the gateway nodes.

Whenever a node generates a request to transfer the data to a particular node, it checks the destination node address in its member table. If the matching node is found in the member table, packet is transferred to that node. If no match is found, then the data packet will be sent to cluster-head. Cluster-head will again check for the match in its member table. If no match is found, cluster-head will check for the node in the Gateway node table at which the required bandwidth is available. The data packet is sent to the node at which the required bandwidth is available. The node address will be copied to List_of_Visited_Nodes field of data packet header. This field will help in the prevention of loops. Using this field, same data packet will not be sent to a particular node more than once. Reduce the available bandwidth of the gateway node. This process will continue till the destination node is reached or if the count of visited nodes get increased than the count in TTL (Time to live) field. If this count becomes more than TTL the data packet is dropped and a message is sent to source node. And finally to ensure that the packets are received in the destination and when the nodes haven't bandwidth desired by the Source, the node stop traffic for a few minutes for complete a management of the queue to avoid packet loss.

\section{SIMULATION EXPERIMENTS}

The proposed protocol has been implemented in the network simulator ns-2 version 2.34 [14]. The IEEE 802.11 DCF (Distributed Coordinated Function) MAC was used as the basic for the experiments with a channel capacity of $2 \mathrm{Mb} / \mathrm{sec}$. 
International Journal of Wireless \& Mobile Networks (IJWMN) Vol. 4, No. 5, October 2012

The transmission range of each node was set to $250 \mathrm{~m}$. CBR is the traffic sources. The number of nodes changed with 3 values $(20,40$ and 60$)$. The simulation parameters are presented in Table 5.

In our proposed model, we chose a topology where there exist fixed nodes that represent Mesh Routers (MR) theses nodes can be $\mathrm{CH}$ or Gateway and mobile nodes that have a randomly circulating, theses node representing Mesh Clients MC as shown figure2.

Three metrics evaluated our network performances, theses metrics are: Packet Delivery Ratio (PDR), Average End to End delay (Delay) and routing Overhead (Overhead).

\begin{tabular}{|c|c|}
\hline Parameters & value \\
\hline Transmission range & $250 \mathrm{~m}$ \\
\hline Propagation channel frequency & $2.4 \mathrm{Ghz}$ \\
\hline Simulation Time & $640 \mathrm{~s}$ \\
\hline Topology size & $1200 \mathrm{~m}^{*} 1000 \mathrm{~m}$ \\
\hline Phy and MAC Model & 802.11 \\
\hline Interface of queue type & PriQueue \\
\hline Antenna & OmniAntenna \\
\hline Cross traffic type & CBR UDP \\
\hline Mobile Nodes Placement & Random \\
\hline Number of Nodes & $20,40,60$ \\
\hline
\end{tabular}

\section{ANALYSIS RESULTS AND DISCUSSION}

The simulation results are shown in the following section in the form of line graph. Graphs show comparison between three protocols (AODV, CBRP and Q-CBRP) by varying different number of mesh clients on the basic of the above mentioned metrics as function of pause time.

\subsection{Packet Delivery Ration (PDR)}

Fig: 4, 5, 6, shows a comparison between the routing protocols on the basic or PDR as a function of pause time and using different number clients mesh. PDR describes the loss rate as seen by the transport layer. According to the graphs, it is clear that PDR decrease with increase in number of clients.

The giver graph shows that Q-CBRP and CBRP performs better in delivering packets which is $93 \%$ and $55 \%$ but AODV shows an average PDR equals to $70 \%$. Between CBRP and QCBRP, Q-CBRP gives slightly better throughput for a larger network size and better scalability comes from its largely reduced flooding for route discovery. 
International Journal of Wireless \& Mobile Networks (IJWMN) Vol. 4, No. 5, October 2012

We can say that the clustering approach provides more interesting PDR ration compared to the basic protocols like AODV because of diminished transmission of messages controls

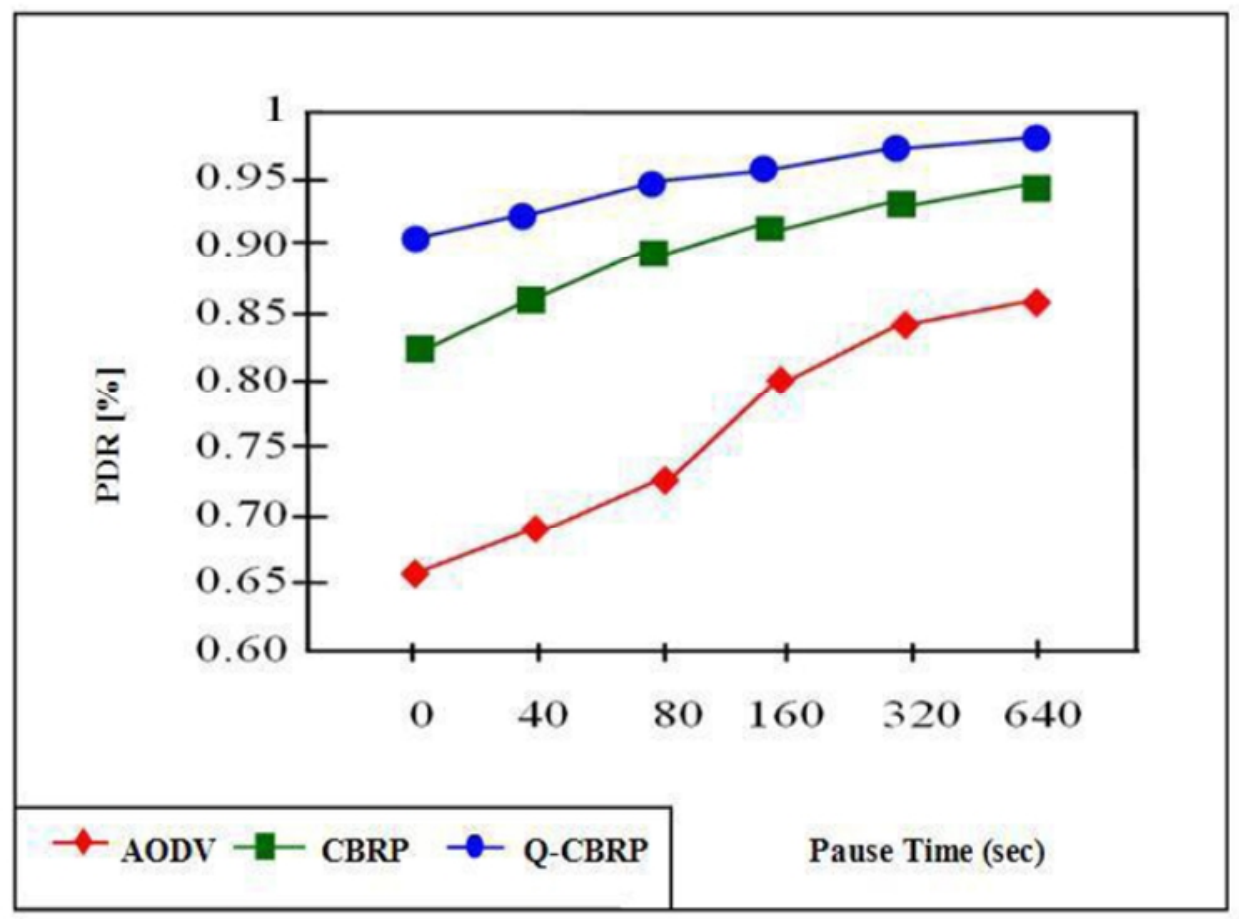

Figure 4. PDR vs pause time for 20 nodes

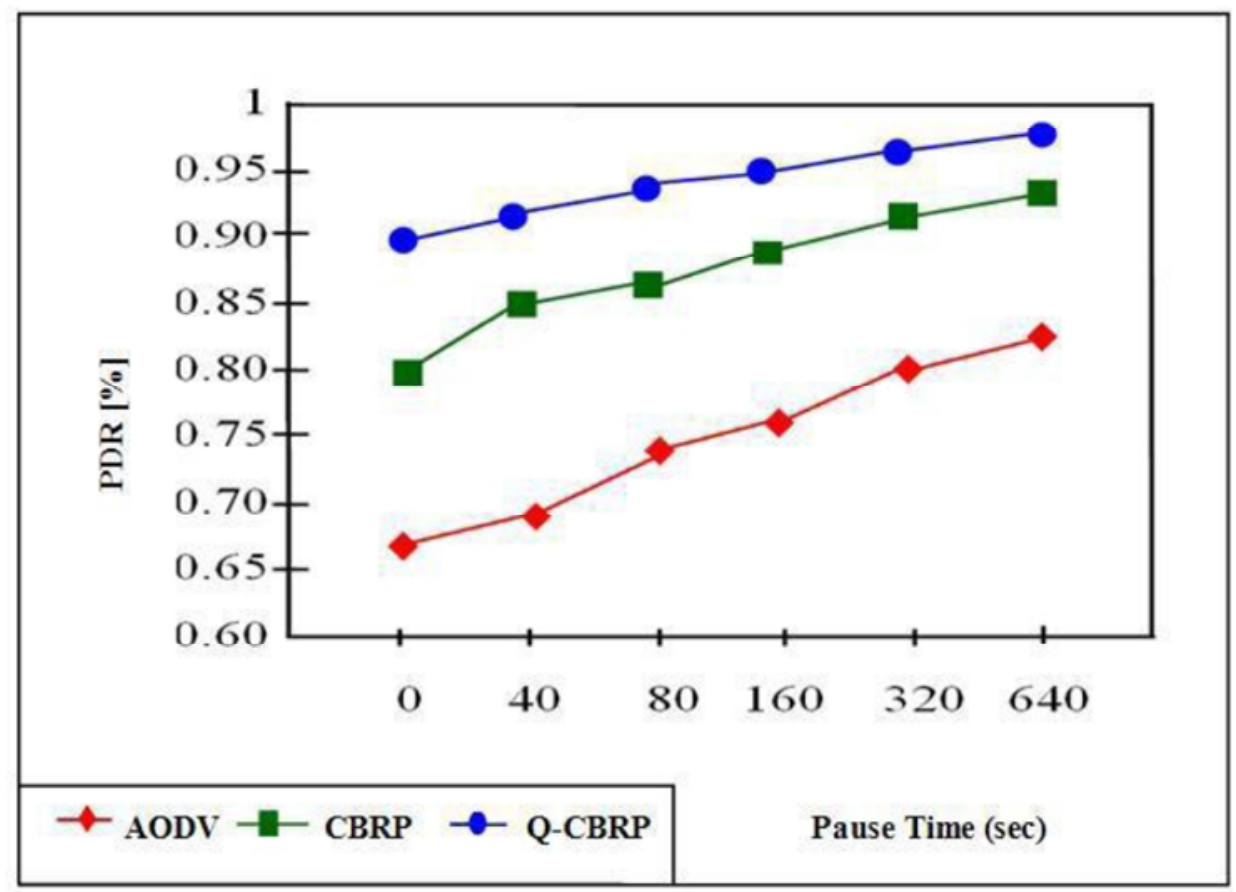

Figure 5. PDR vs pause time for 40 nodes 


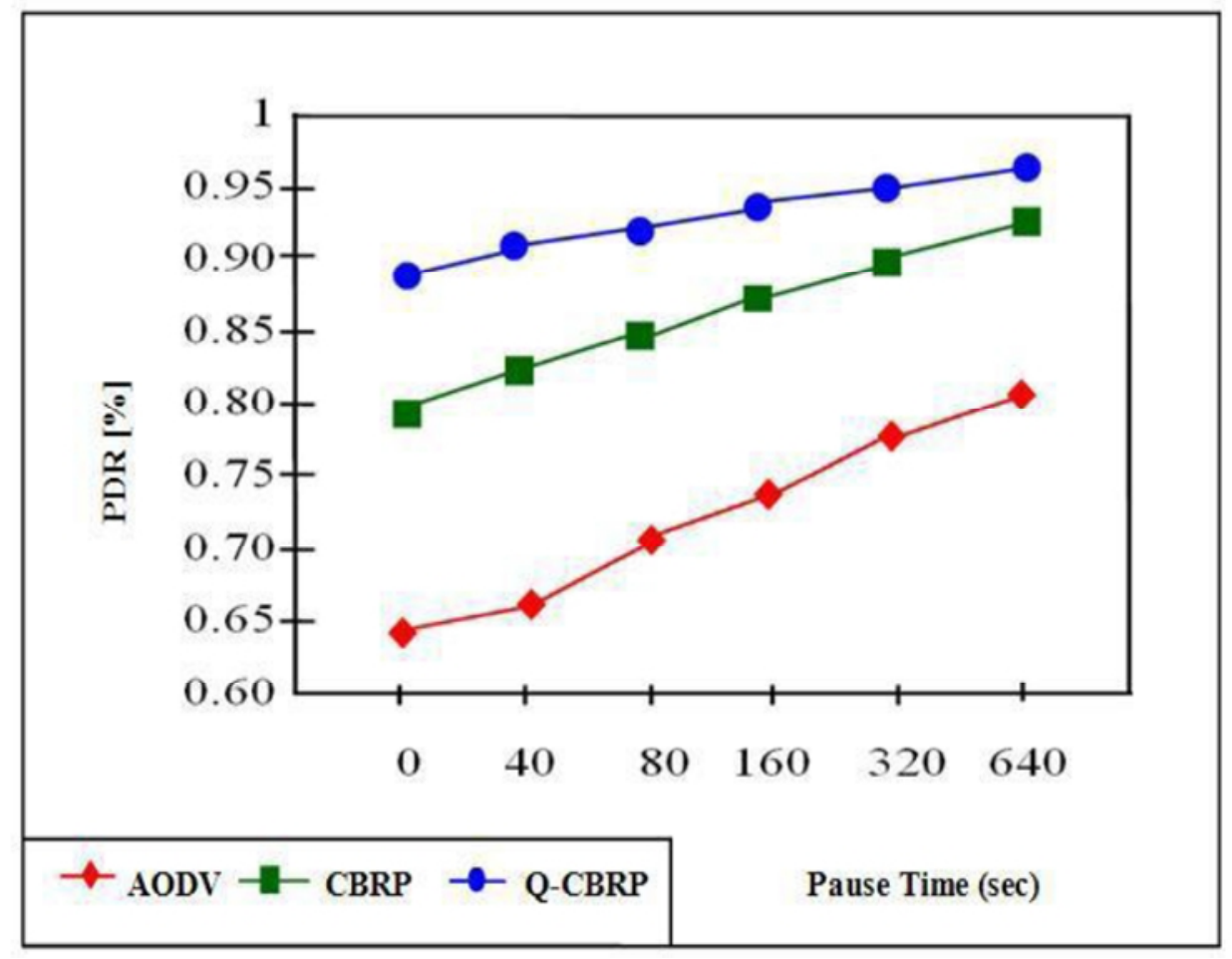

Figure 6. PDR vs pause time for 60 nodes

\subsection{Average End to End Delay}

Fig: 7, 8, 9, shows the graphs for end to end delay versus pause time. From these graphs we see that the average packet delay increase for increase in number of nodes waiting in the interface queue while routing protocols try to find valid route to the destination. Besides the actual delivery of data packets, the delay time is also affected by route discovery, which is the first step to begin a communications session. The source routing protocols have a longer delay because their route discovery takes more time as every intermediate node tries to extract information before forwarding the reply. The same thing happens when a data packet is forwarder hop by hop. Hence while source routing makes route discovery more profitable, it slows down the transmission of packets.

Out of the tree routing protocols, Q-CBRP has the shortest average end to end delay. Then CBRP and AODV.

The AODV protocol is already the best which provide End To End delay in a mesh network following previous researchs; In our case the use of a clustering approach, due to communications between Cluster Head $(\mathrm{CH})$ and gateway and only between $\mathrm{CH}$ and mesh users, these facts reduced the network load and automatically improves Delay in Mesh networks 
International Journal of Wireless \& Mobile Networks (IJWMN) Vol. 4, No. 5, October 2012

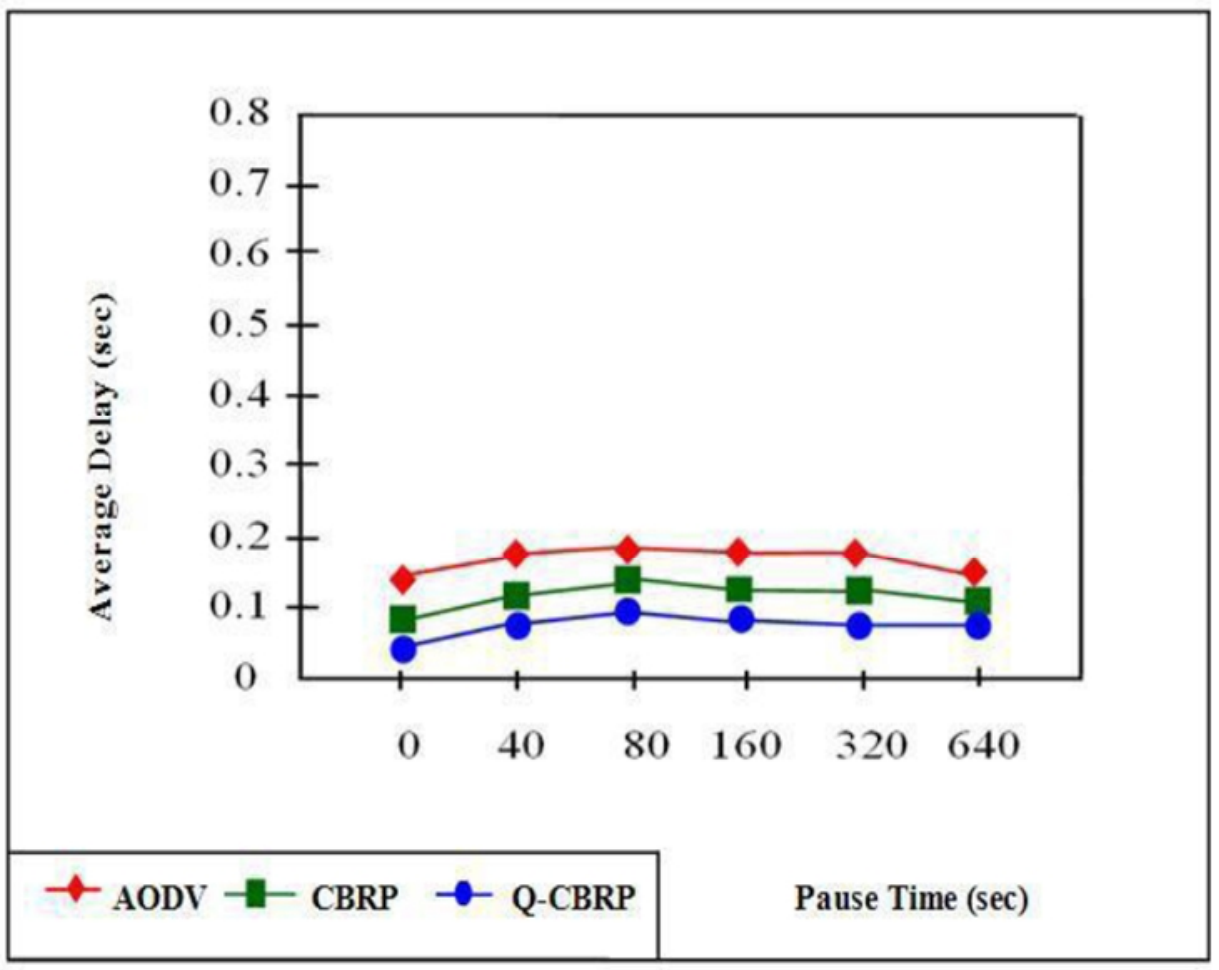

Figure 7. Average End-to-End Delay vs pause time for 20 nodes

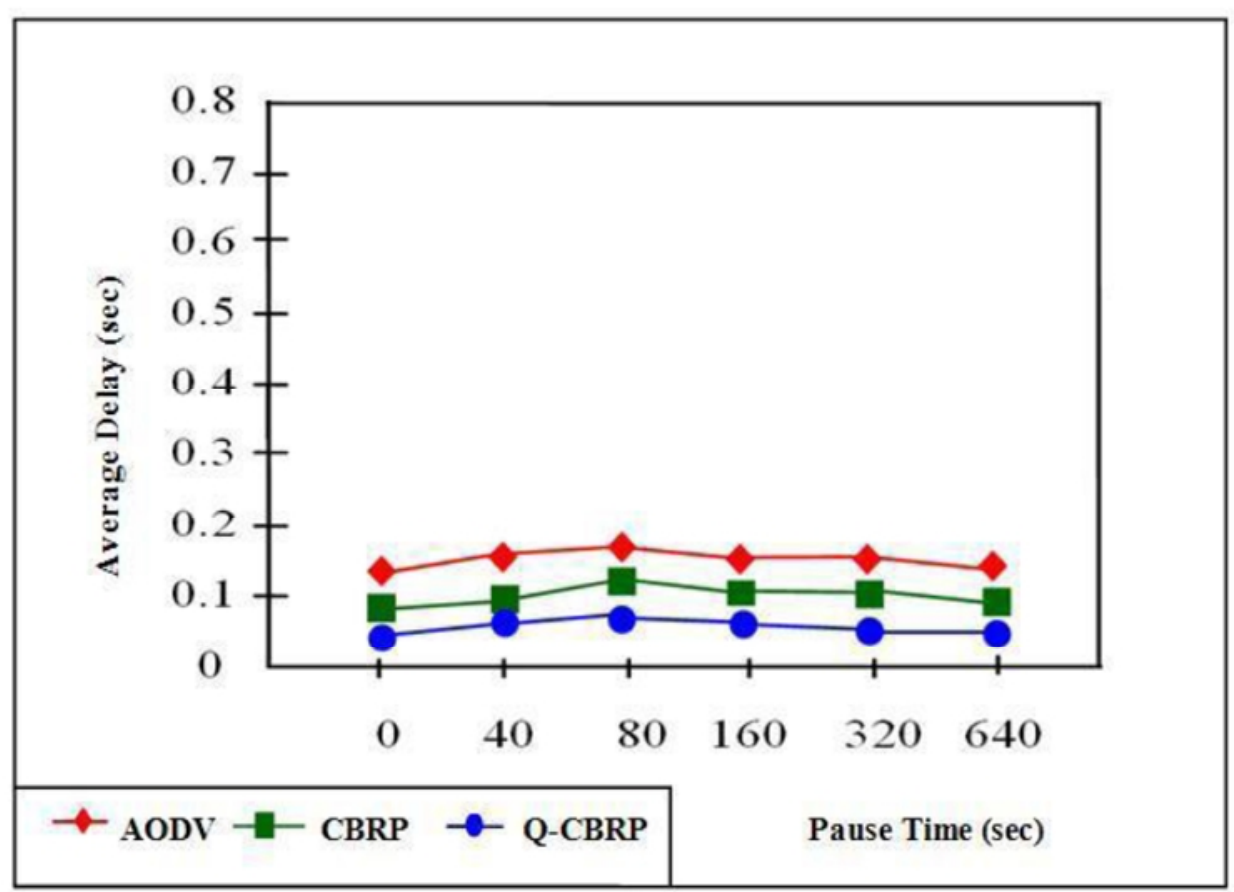

Figure 8. Average End-to-End Delay vs pause time for 40 nodes 


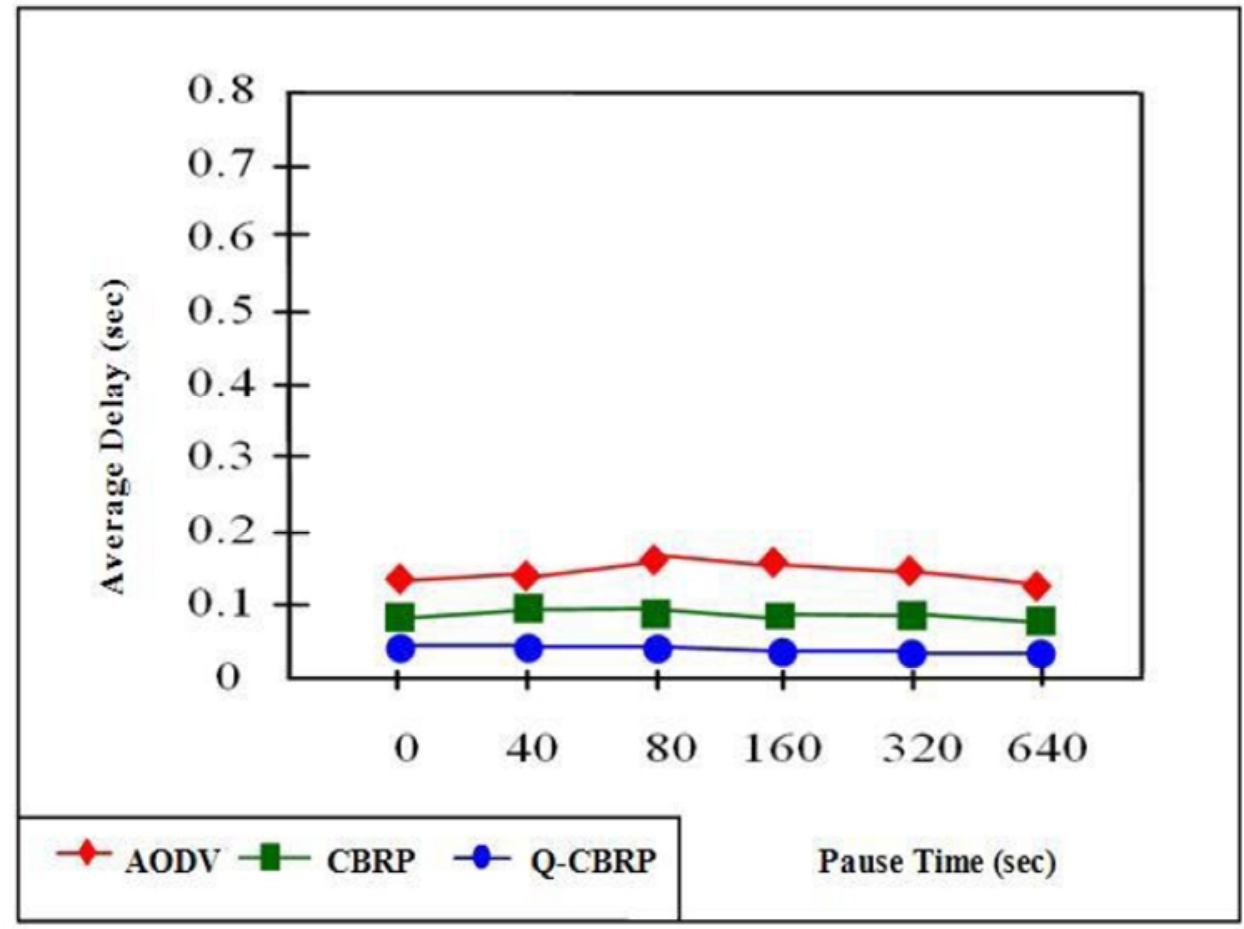

Figure 9. Average End-to-End Delay vs pause time for 60 nodes

\subsection{Routing Overhead}

Fig: 10, 11, 12, shows the performance of Q-CBRP, CBRP and AODV by evaluating packet overhead with varying pause time. Average packet overhead per packet received is 1.45, 2.41 and 3.20 for Q-CBRP, CBRP and AODV respectively.

These results due to a smaller flooding range of Q-CBRP and CBRP and route requests and replies are very less. But message Hello of a clustering algorithm can be larger than the size of the Hello messages of AODV. Hence these three protocols have substantially the same overhead rate when numbers of nodes increase but clustering algorithms are still the best. 
International Journal of Wireless \& Mobile Networks (IJWMN) Vol. 4, No. 5, October 2012

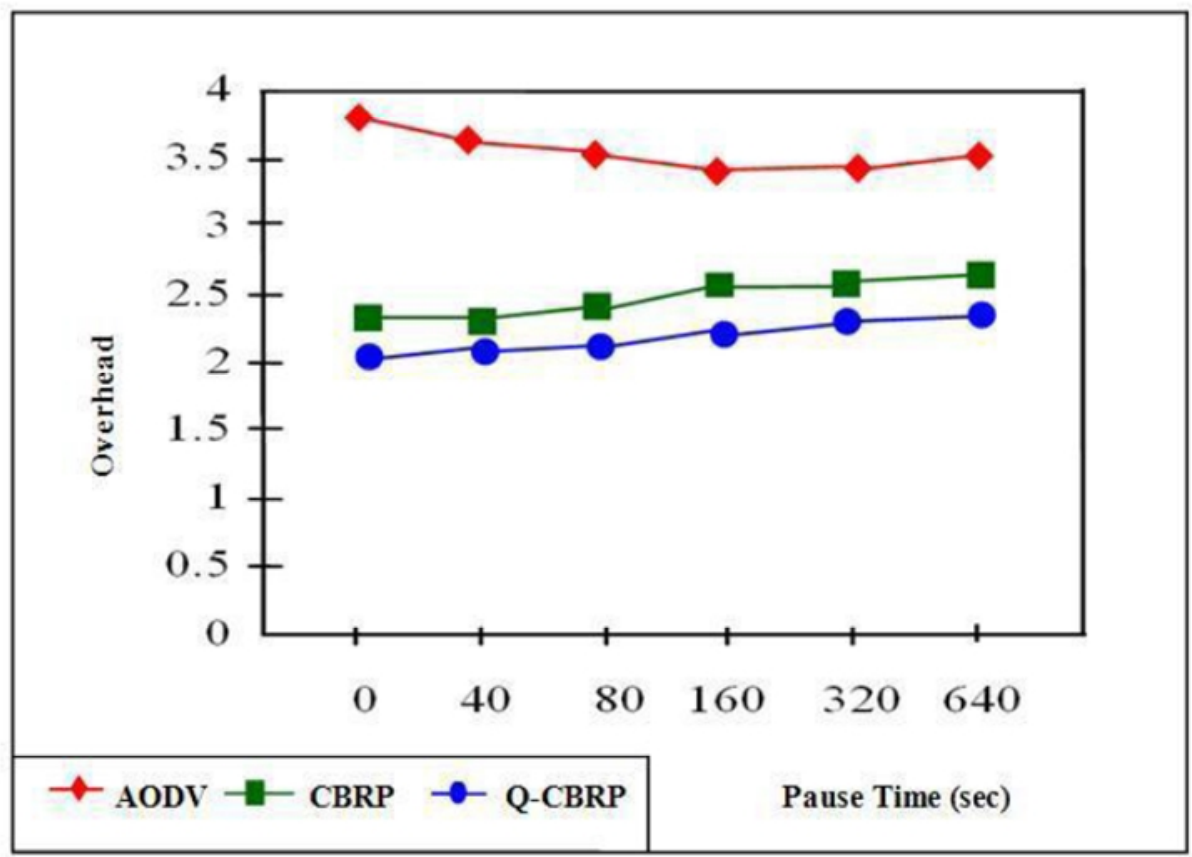

Figure 10. Overhead vs pause time for 20 nodes

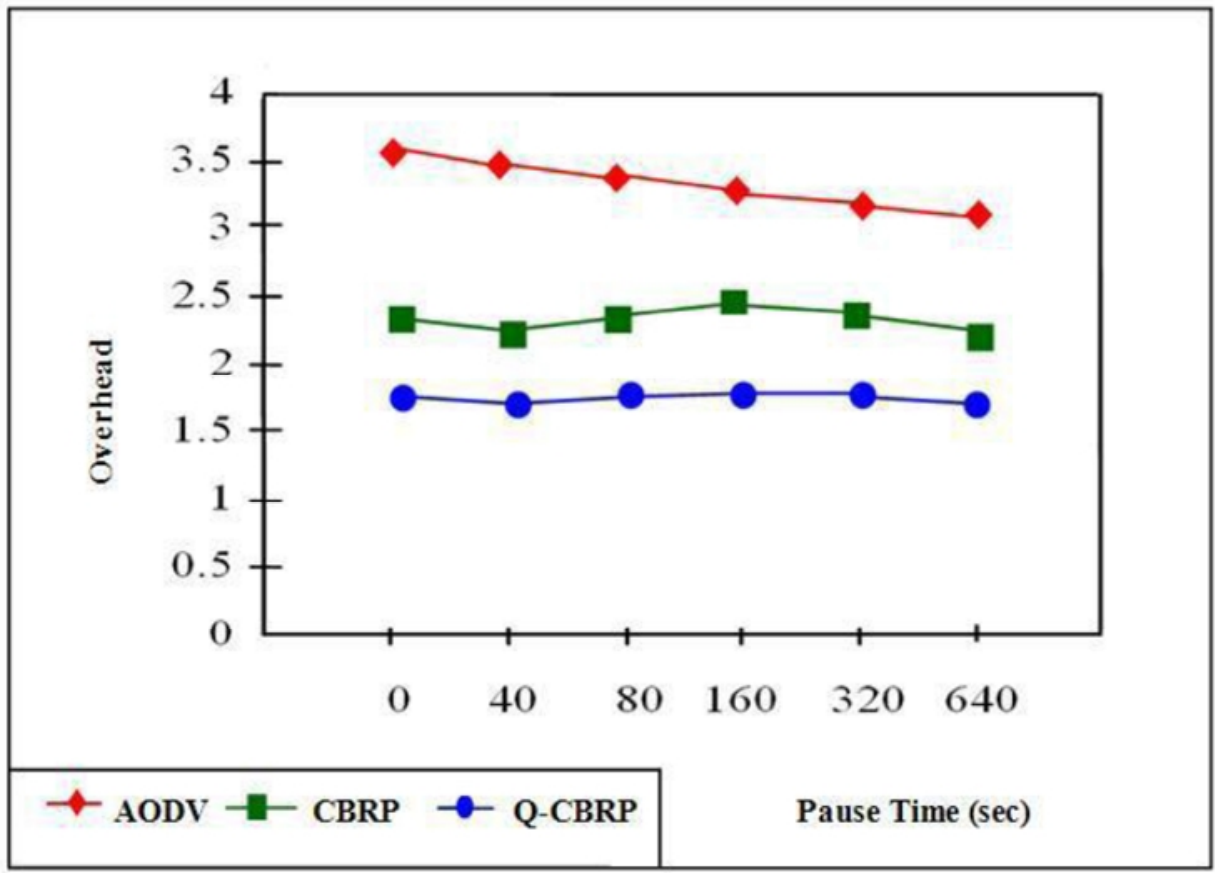

Figure 11. Overhead vs pause time for 40 nodes 


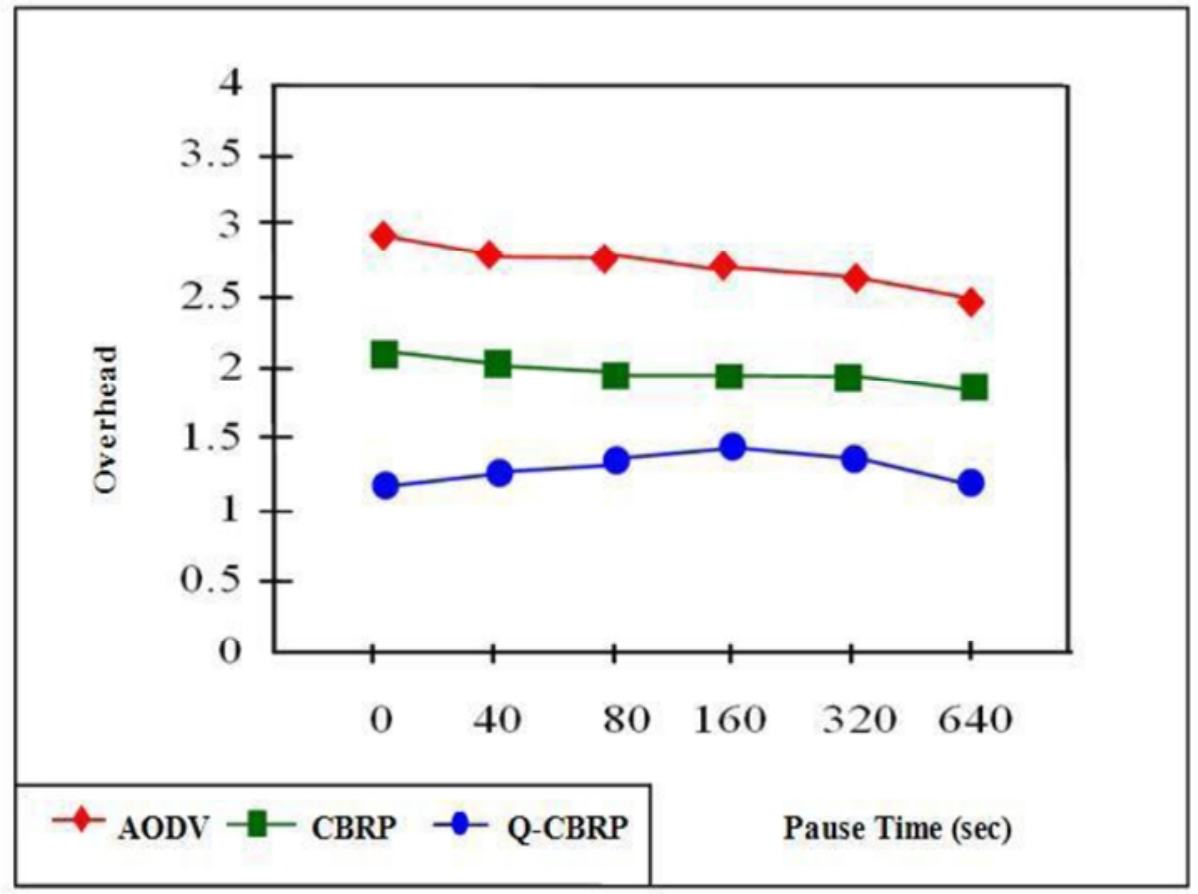

Figure 12. Overhead vs pause time for 40 nodes

\section{CONCLUSION AND PERSPECTIVE}

This paper compared the performance of our Algorithm Q-CBRP in WMN and others two routing protocols used in WSN, Ad-hoc and MANETs network (CBRP and AODV). These routing protocols were compared in terms of Packet delivery ratio, Average delay and routing overhead when subjected to change in pause time and varying number of Mesh clients. The results show that by comparing the performance between Q-CBRP, CBRP and AODV, we can conclude that cluster topologies bring scalability and routing efficiency for a WMN as network size increase. By adding the management of bandwidth to our own algorithm with admission control, and add some

filed in Data header plus some modification on routing Table, the mesh network is able to transport multimedia streams by offering a wider and more stable throughput compared to the basic protocol (CBRP).

For Further research we plan to use a random mobility for Mesh clients, we will specified the type of traffic (HTTP, FTP and Video Streaming) and measure the different criteria of QoS in a mobile WMN.

\section{REFERENCES}

[1] Ian F. Akyildiz And XudongWang "Wireless Mesh Networks", Edition : WILEY , 2009.

[2] Jaydip Sen "A Throughput Optimizing Routing Protocol for Wireless Mesh Networks". 12th IEEE International Conference on High Performance Computing and Communications.2010 
International Journal of Wireless \& Mobile Networks (IJWMN) Vol. 4, No. 5, October 2012

[3] S. Y. Hui, K. H. Yeung, K. Y. Wong, “ Optimal Placement of Mesh Points in a Wireless Mesh Network Using Multipath Routing Protocol”, . International Conference on Network and Parallel Computing. IEEE Computer society.2008.

[4] Anna Zakrzewska, Leszek Koszalka, Iwona Pozniak-Koszalka, Andrzej Kasprzak "Analysis of Routing Protocol Performance in Wireless Mesh Networks" International Conference of Computational Science and Its Applications, 2010

[5] R. Braden, D. Clark, and S. Shenker, "Integrated services in the Internet architecture: an overview," 1994, IETF RFC 1633.

[6] S. Blake, D. Black, M. Carlson, E. Davies, Z.Wang, andW. Weiss, "An architecture for differentiated services," 1998, IETF RFC 2475.

[7] Yinpeng Yu, Yuhuai Peng, Lei Guo, Xingwei Wang, "Performance Evaluation for Routing Protocols in Wireless Mesh Networks", International Coriference on Educational and Information Technology Performance, 2010 .

[8] Yong Zhang, Yifei Wei, Mei Song, Junde Song "R-AODV Rate Aware Routing Protocol for Wifi Mesh Network" ICWMMN2006.

[9] Bhupendra Kumar Gupta and B.M.Acharya Manoj Kumar Mishra "Optimization of routing algorithm in Wireless Mesh Network" IEEE, 2009

[10] Jaydip Sen “A Throughput Optimizing Routing Protocol for Wireless Mesh Networks”. 12th IEEE International Conference on High Performance Computing and Communications.2010.

[11] Rainer Baumann, Simon Heimlicher, Vincent Lenders, Martin May "Routing Packets into Wireless Mesh Networks" Third IEEE International Conference on Wireless and Mobile Computing, Networking and Communications (WiMob 2007).

[12] Chungui Liu, Yantai Shu and Lianfang Zhang, Maode Ma "Backup Routing for Multimedia Transmissions over Mesh Networks" 2007 IEEE

[13] Performance Comparison of MANETs Routing Protocols for Denseand Sparse Topology Mohammed A. Mahdi and Tat-Chee Wan International Conference on Information and Computer Networks (ICICN 2012)

[14] Mukesh Kumar,Rahul Rishi,D.K. Madan "Comparative Analysis of CBRP,DSR, AODV Routing Protocol in MANET, IJCSE. 2010

[15] Tim Daniel Hollerung, “The Cluster-Based Routing Protocol”, University of Paderborn, 2004.

[16] www.isi.edu/nsnam/ns/ Network simulator ns-2, 2009 